\title{
Current Mode Neutron Noise Measurements in the Zero Power Reactor CROCUS
}

\author{
O. Pakari, V. Lamirand, G. Perret, L. Braun, P. Frajtag, A. Pautz
}

\begin{abstract}
The present article is an overview of developments and results regarding neutron noise measurements in current mode at the CROCUS zero power facility. Neutron noise measurements offer a non-invasive method to determine kinetic reactor parameters such as the prompt decay constant at criticality $\alpha=\beta_{\text {eff }} / \Lambda$, the effective delayed neutron fraction $\beta_{\text {eff }}$, and the mean generation time $\Lambda$ for code validation efforts. At higher detection rates, i.e. above $2 \times 10^{4}$ cps in the used configuration at $0.1 \mathrm{~W}$, the previously employed pulse charge amplification electronics with $\mathrm{BF}_{3}$ detectors yielded erroneous results due to dead time effects. Future experimental needs call for higher sensitivity in detectors, higher detection rates or higher reactor powers, and thus a generally more versatile measurement system. We, therefore, explored detectors operated with current mode acquisition electronics to accommodate the need. We approached the matter in two ways:
\end{abstract}

1) By using the two compensated ${ }^{10} \mathrm{~B}$-coated ionization chambers available in CROCUS as operational monitors. The compensated current signal of these chambers was extracted from core monitoring output channels.

2) By developing a new current mode amplification station to be used with other available detectors in core. Characteristics and first noise measurements of the new current system are presented. We implemented post-processing of the current signals from 1) and 2) with the APSD/CPSD method to determine $\alpha$. At two critical states $(0.5$ and $1.5 \mathrm{~W})$, using the ${ }^{10} \mathrm{~B}$ ionization chambers and their CPSD estimate, the prompt decay constant was measured after 1.5 hours to be $\alpha=(156.9 \pm 4.3) \mathrm{s}^{-1}(1 \sigma)$. This result is within $1 \sigma$ of statistical uncertainties of previous experiments and MCNPv5-1.6 predictions using the ENDF/B-7.1 library. The new system connected to a CFUL01 fission chamber using the APSD estimate at $100 \mathrm{~mW}$ after $33 \mathrm{~min}$ yielded $\alpha=(160.8 \pm 6.3) \mathrm{s}^{-1}$, also within $1 \sigma$ agreement.

The improvements to previous neutron noise measurements include shorter measurement durations that can achieve comparable statistical uncertainties and measurements at higher detection rates.

Index Terms - Research reactors, neutron noise, current acquisition, kinetic parameter measurement

Transcript received $1^{\text {st }}$ June 2017

O. Pakari, L. Braun, and P. Frajtag are with the LRS, EPFL (Corresponding author: O. Pakari, email: oskari.pakari@epfl.ch). G. Perret is with the LRS, Paul Scherrer Institut (PSI). V. Lamirand and A. Pautz are both with the LRS, EPFL and NES, PSI.

\section{INTRODUCTION}

$\mathrm{N}$ EUTRON noise measurements in zero power reactors provide the means to determine kinetic parameters such as the prompt neutron decay constant $\alpha$, the delayed neutron fraction $\beta_{\text {eff }}$, and the mean prompt generation time $\Lambda$ in a noninvasive manner. These values then contribute to code validation and nuclear database efforts.

The kinetic parameters of the CROCUS zero power reactor core have been successfully determined using reactivity change [1] and non-invasive neutron noise measurements with the Feynman- $\alpha$ and auto and cross power spectral density (APSD/CPSD) methods [2, 3]. The measurement station was also successfully used in the MINERVE zero power facility [4]. The previous measurements in CROCUS were undertaken with several sub-critical and critical states with powers up to $0.1 \mathrm{~W}$ with four $\mathrm{BF}_{3}$ neutron detectors in the control rod and peripheral fuel rod positions (see Fig. 2). Measurements at higher power or with more sensitive detectors could not be performed with the developed measurement station based on pulse spectrometry electronics due to pile-up in the detector and dead time effects in the amplification stage. Future experimental aspirations in CROCUS [5] call for more sensitivity, higher precision, and extended versatility in detector positioning. For this purpose, we examined the option of using current measurement electronics for neutron noise measurements in CROCUS. This paper is dedicated to describing the latest developments, beginning with an introduction to the CROCUS facility in Section II and a detailed description of the used instrumentation. In Section III, we present the measurement of the kinetic parameters using the available current signal of core monitors. The measurement setup alongside the APSD/CPSD analysis method with associated post processing details and results are discussed. In Section IV we outline the development and first noise measurements of a novel current amplifier used with Photonis CFUL01 fission chambers ( $\left.1 \mathrm{~g}{ }^{235} \mathrm{U}\right)$. This is followed by an outlook on future developments.

\section{THE RESEARCH REACTOR CROCUS}

\section{A. Reactor facility description}

The CROCUS reactor is a two-zone, uranium-fueled, $\mathrm{H}_{2} \mathrm{O}$ moderated facility operated by the Laboratory for Reactor Physics and Systems Behaviour (LRS) at the Swiss Federal Institute of Technology Lausanne (EPFL) (see Fig. 1). It is a 


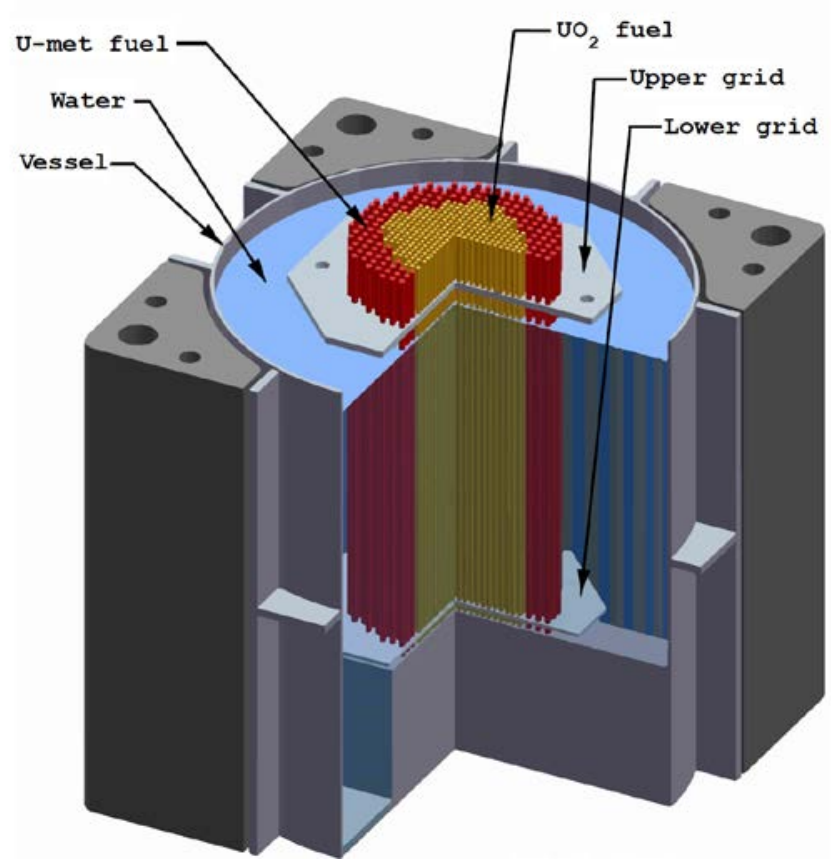

Fig. 1. The CROCUS reactor vessel with basic internal structures. Two fuel zones, an inner $\mathrm{UO}_{2}$ and outer $\mathrm{U}_{\text {met }}$ zone, held in an approximately cylindrical array by two grids. Water is filled from the bottom to establish criticality.

zero power reactor, with a nominal power of up to $100 \mathrm{~W}$. The core is approximately cylindrical in shape with a diameter of about $58 \mathrm{~cm}$ and a height of $100 \mathrm{~cm}$. The core reactivity is controlled by a variation of the water level with an accuracy of $0.1 \mathrm{~mm}$ (equivalent to $0.4 \mathrm{pcm}$ ) and/or by means of two control rods containing naturally enriched boron carbide $\left(\mathrm{B}_{4} \mathrm{C}\right)$ sintered pellets located symmetrically within the outer fuel zone. Two different kinds of fuel rods make up the reactor core of CROCUS (see Fig. 1 and 2). The central zone is loaded with $336 \mathrm{UO}_{2}$ fuel rods (1.806 wt.\%-enriched), set in a square lattice with a pitch of $1.837 \mathrm{~cm}$. The peripheral zone is loaded with up

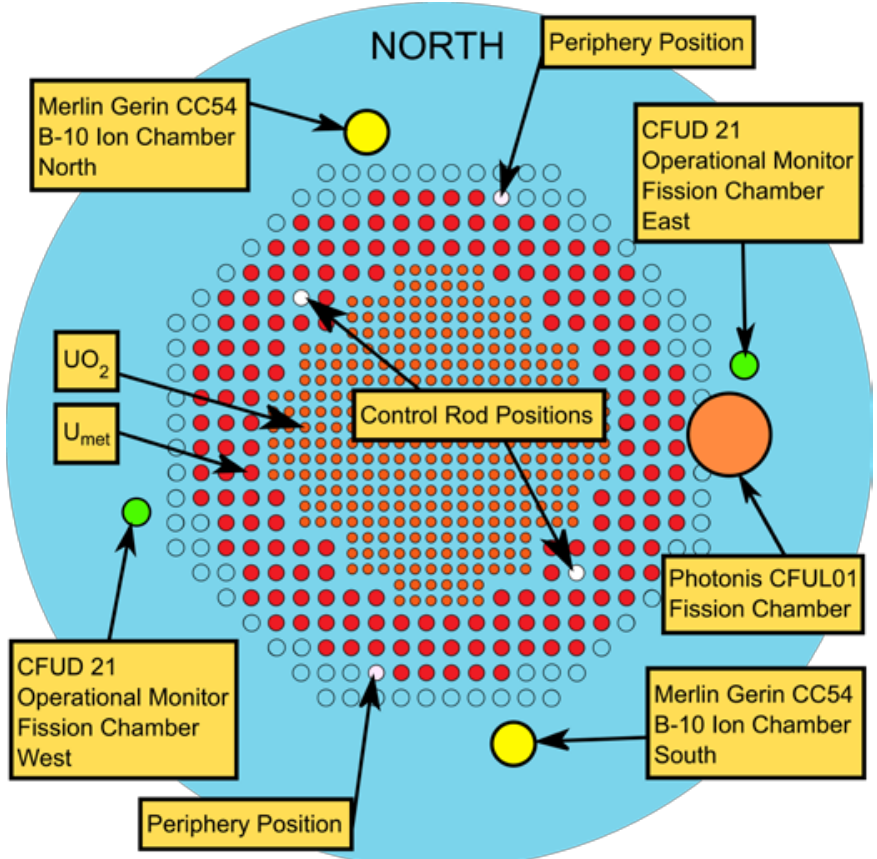

Fig. 2. Top view of the CROCUS reactor core with detector locations used for noise measurements and core monitoring. to 176 thicker, $\mathrm{U}_{\text {met }}$ fuel rods $(0.947 \mathrm{wt} . \%$-enriched $)$ with a pitch of $2.917 \mathrm{~cm}$, also in a square lattice. All fuel rods have an aluminum cladding and are maintained in a vertical position by the upper grid and lower grid plates spaced $100 \mathrm{~cm}$ apart. Both grid plates incorporate a cadmium layer with a thickness of $0.5 \mathrm{~mm}$ to limit axial thermal leakage to surrounding structures. The active fuel length starts at the top surface of the lower cadmium layer and extends to $100 \mathrm{~cm}$. The core is located in an aluminum water tank, its diameter being $130 \mathrm{~cm}$ and thickness being $1.2 \mathrm{~cm}$. Demineralized light water $\left(\mathrm{H}_{2} \mathrm{O}\right)$ is used as moderator and reflector.

\section{B. Core instrumentation}

CROCUS is equipped with four operational monitor detectors. Two Merlin Gerin CC54 Compensated ${ }^{10} \mathrm{~B}$ coated Ionisation Chambers (CIC north \& CIC south) with a sensitivity of $3 \times 10^{-5} \mathrm{nA} / \mathrm{n}_{\text {th }}$ are used in current mode for flux control and core monitoring. The CICs have an active length of $355 \mathrm{~mm}$ and diameter of $49 \mathrm{~mm}$. Two Photonis CFUD 21 fission chambers (FC) in the eastern and western periphery serve as the operational monitors and are operated in pulse mode. They are part of the safety channels and are calibrated with respect to reactor power with gold foil irradiation experiments, and are, thus, reactor power monitors (see Fig. 2). The readout of the fission chamber monitors is used for fission rate $F_{0}$ determination via

$$
F_{0}=\overline{C_{\text {mon }}} \kappa / E,
$$

with $\overline{C_{\text {mon }}}$ being the average monitor count rate in cps during the experiment, $E$ being the energy released per fission in $\mathrm{J}$ and $\kappa^{-1}=2428.0 \pm 96 \mathrm{cps} / \mathrm{W}$ being the calibration factor for the western chamber. $\kappa$ was determined using eight gold foils irradiated in the center of the core with even axial spacing at $15 \mathrm{~W}$ for 1 hour. The effective cross sections for the gold foils were calculated using MCNP5-v1.6 with the ENDF/B-7.1 library.

\section{Pulse mode neutron noise measurement station}

The established neutron noise measurement station consists of four $\mathrm{BF}_{3}$ neutron detectors in control rod and peripheral fuel rod positions connected to standard gamma spectrometry preamplifiers and amplifiers (see Fig 2.). The pulses are then read out via Multi Channel Scaler cards on a computer. The system is currently limited to measurements at sub-criticality or critical states with powers below $0.1 \mathrm{~W}$ at $18 \mathrm{kcps}$ due to pileup in the detector and dead time effects in the amplifying stage.

\section{Limitations and motivation for current mode measurement}

Neutron noise measurements generally require sensitive detectors to minimize detector shape or other spatial effects [6]. The $\mathrm{BF}_{3}$ detector has a sensitivity of $4.7 \times 10^{-2}$ counts $/ \mathrm{n}_{\text {th }}$ with an active length of $100 \mathrm{~mm}$ and a $7.5 \mathrm{~mm}$ diameter. Other available detectors such as Vniitfa ${ }^{3} \mathrm{He}$ ionization chambers (2.82 counts $/ \mathrm{n}_{\mathrm{th}}, 340 \mathrm{~mm}$ active length, $32 \mathrm{~mm}$ diameter) and Photonis CFUL01 fission chambers ( 1 count $/ \mathrm{n}_{\text {th }}, 211 \mathrm{~mm}$ active length, $48 \mathrm{~mm}$ diameter) are too sensitive for in-core usage with the existing electronics, even at low powers or sub-critical states. Thus to increase our flexibility in detector choice and 
ultimately detector position in CROCUS, we decided to investigate the option to use current mode acquisition. The integration of charge eliminates dead time effects and extends the effective utility range in terms of power [7]. This has been successfully implemented by CEA in the zero power facilities MINERVE and EOLE using an in-house developed system [11] to measure the kinetic parameters. A cross-comparison measurement campaign between this system, SCK $\cdot \mathrm{CEN}$ and PSI/EPFL stations was undertaken within the Venus-EoleProteus scientific cooperation and will be reported on soon.

In the following sections we present two approaches to examine the feasibility of current mode measurements for neutron noise applications in CROCUS: firstly, by directly measuring the already available current output of the CIC monitors using an advanced oscilloscope and by applying noise analysis techniques to determine the kinetic parameters. This is compared to previous results to verify the validity of the new method. Secondly, by showing the development status of a new current amplification and measurement system, to be used for various detectors, that allows for flexible detectors and detector location choice.

\section{Measurement of the Kinetic Parameter $\alpha$ in CURRENT MOde In CROCUS USING CORE MONITORS}

\section{A. Acquisition of CIC signals}

We used two higher reactor power configurations $(0.5 \mathrm{~W}$ and $1.5 \mathrm{~W}$ ) for the current mode acquisition (see Table I) to show the increased capability in terms of detection rates compared to the previous $\mathrm{BF}_{3}$ setup. Calculations showed an order of magnitude higher detection rate in the CFUL01 fission chamber in the periphery compared to $\mathrm{BF}_{3}$ detector in the control rod position. The acquisition line is depicted in Fig. 3. Both CIC signals were directly connected to the $10 \mathrm{M} \Omega \mathrm{DC}$ inputs of a Teledyne Lecroy Wavesurfer 10 oscilloscope [12]. At a sampling rate of $10 \mathrm{kHz}$ the acquisition lasted up to 33 min per measurement. We measured three consecutive times to achieve a total measurement duration of about 1.5 hours at both reactor powers.

\section{B. Power spectral density noise analysis}

The auto or cross power spectral density (APSD/CPSD) analysis method, often referred to as the Cohn- $\alpha$ method [7], is

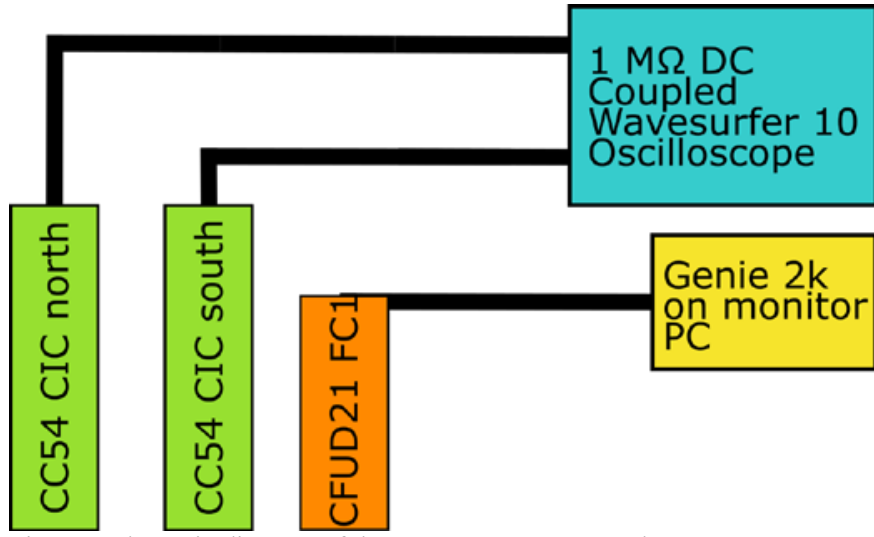

Fig. 3. Schematic diagram of the measurement setup. The two CICs are used in current mode for noise measurements whilst the calibrated CFUD21 fission chamber 1 is used for fission rate determination.
TABLE I

USED REACTOR CONFIGURATIONS FOR NEUTRON NOISE MEASUREMENTS

\begin{tabular}{llll}
\hline \hline $\begin{array}{l}\text { Power } \\
(\mathrm{W})\end{array}$ & $\begin{array}{l}\text { Count rate of FC 1 } \\
\text { monitor }(\mathrm{cps})\end{array}$ & $\begin{array}{l}\text { Mean voltage } \\
\text { of CIC }(\mathrm{V})\end{array}$ & Fission rate $\left(\mathrm{s}^{-1}\right)$ \\
\hline 0.5 & 1229 & 1.63 & $1.57 \mathrm{E}+10$ \\
1.5 & 3904 & 5.29 & $5.00 \mathrm{E}+10$
\end{tabular}

the most common method used for current mode signals. By calculating the Fourier transform of auto- and cross-correlation functions of a detector signal, the reactor noise at criticality can be fitted by the following general expression in dependency of the angular frequency $\omega[4,8,9,10]$ :

$$
G_{i j}(\omega)=\frac{2 S_{i} S_{j} D_{v}}{\Lambda^{2} F_{0}} \frac{1}{\omega^{2}+\alpha^{2}}+S_{i} S_{j} \delta_{i j}
$$

with $\delta_{i j}$ being the Kronecker delta, and $S_{i}$ the measured detector signal. $D_{v}$ is the Diven factor [13], calculated for CROCUS in [3] to be 0.806 . From a lumped parameter perspective white noise is transferred through a low-pass filter with cut-off frequency at $f_{0}=\alpha / 2 \pi$. The plateau at frequencies much smaller than $f_{0}$ are proportional to the effective delayed neutron fraction $\beta_{\text {eff. }}$

The calculation of the one-sided APSD and CPSD was implemented in a MATLAB script using the Welch overlapped segment estimator method. A Hann window with $50 \%$ overlap was chosen for smoothing [14] and 24,000 total discrete Fourier transform points to allow for a $0.5 \mathrm{~Hz}$ resolution. As the oscilloscope had a sampling frequency of $10 \mathrm{kHz}$, the bandwidth of the PSD is $2500 \mathrm{~Hz}$.

Code predictions for the prompt decay constant using MCNP5v1.6 with the ENDF/B-7.1 library yielded $\alpha=(158.6 \pm 1.6) \mathrm{s}^{-1}$, hence an expected cut-off frequency of $f_{0}=\alpha / 2 \pi=25.2 \mathrm{~Hz}$. Thus the region of interest for curve analysis was limited to $100 \mathrm{~Hz}$. For this region of the APSD or CPSD a fit function of the shape

$$
G_{i j}(\omega)=\frac{a}{\omega^{2}+f_{0}^{2}}+c
$$

was used, according to Equation 2. $c$ is introduced to account for white noise introduced by imperfect electronics and uncorrelated current. For uncertainty estimation the fit data was weighted by the standard deviation, for an APSD/CPSD being

$$
\sigma_{G_{i i}}(\omega)=\frac{G_{i i}}{\sqrt{N_{D F T}}} ; \sigma_{G_{i j}}(\omega)=\sqrt{\frac{G_{i j}(\omega) G_{i j}^{*}(\omega)}{N_{D F T} \gamma_{i j}^{2}(\omega)}}
$$

with $N_{D F T}$ being the number of discrete Fourier transforms and $\gamma_{i j}(\omega)$ being the coherence function between the two detectors,

$$
\gamma_{i j}(\omega)=\frac{\left|G_{i j}(\omega)\right|}{\sqrt{G_{i i}(\omega) G_{j j}(\omega)}},
$$

also calculated using a magnitude squared estimate function in MATLAB.

TABLE II

RESULTS FOR THE PROMPT DECAY CONSTANT AT CRITICALITY

\begin{tabular}{lll}
\hline \hline Power $(\mathrm{W})$ & 0.5 & 1.5 \\
\hline APSD north $\left(\mathrm{s}^{-1}\right)$ & $187.5 \pm 5.2$ & $189.2 \pm 4.6$ \\
APSD south $\left(\mathrm{s}^{-1}\right)$ & $187.2 \pm 4.8$ & $193.2 \pm 4.7$ \\
CPSD $\left(\mathrm{s}^{-1}\right)$ & $156.8 \pm 3.2$ & $160.2 \pm 3.5$
\end{tabular}




\section{Experimental Results for CIC noise measurements}

The APSD and CPSD estimates for the prompt decay constant $\alpha$ based on the previously described measurement and processing setup are presented in Table 2, with example CPSDs with fits and residuals displayed in Fig. 4. The listed uncertainties were calculated by fitting the APSD/CPSD data with differing upper frequency limits. The limits were chosen in $10 \mathrm{~Hz}$ increments between 50 and $100 \mathrm{~Hz}$. The final uncertainty is the propagated uncertainty of the fit uncertainty on $\alpha$ over all five fits. The APSD estimates are subject to transfer function biases which are eliminated by crosscorrelating. The CPSD estimate yields the most accurate value and thus the best estimate using the CICs. The prompt decay constant is found to be $(\mathbf{1 5 6 . 8} \pm \mathbf{3 . 2}) \mathrm{s}^{-1}$ at $0.5 \mathrm{~W}$ and $(\mathbf{1 6 0 . 2} \pm \mathbf{3 . 5}) \mathrm{s}^{-\mathbf{1}}$ at $1.5 \mathrm{~W}$ after 1.5 hours of measurement time, respectively.

D. Comparison to previous measurements and code predictions

With the pulse mode setup two major campaigns were undertaken, the first [2] yielded $\alpha=(\mathbf{1 4 6 . 6} \pm \mathbf{6 . 3}) \mathbf{s}^{-1}$ after 6 to 7 hours of measurement time using the Feynman- $\alpha$ method. A further refined campaign [3] used the APSD/CPSD estimate and found $\alpha=(154.4 \pm \mathbf{2 . 4}) \mathrm{s}^{-1}$ after 5 hours of measurement time. The MCNP prediction of $\alpha=(\mathbf{1 5 8 . 6} \pm \mathbf{1 . 6}) \mathrm{s}^{-1}$ is thus met by both the previous measurements within $2 \sigma$ and $1 \sigma$, respectively. The current mode measurements both agree within $1 \sigma$ with the code predictions. We conclude that current mode measurements in this detection configuration are equally viable for kinetic parameter measurements in CROCUS compared to pulse mode measurements, and similar in terms of measurement time to achieve comparable statistical uncertainties, as the measurements produce a similarly precise value compared to code predictions.
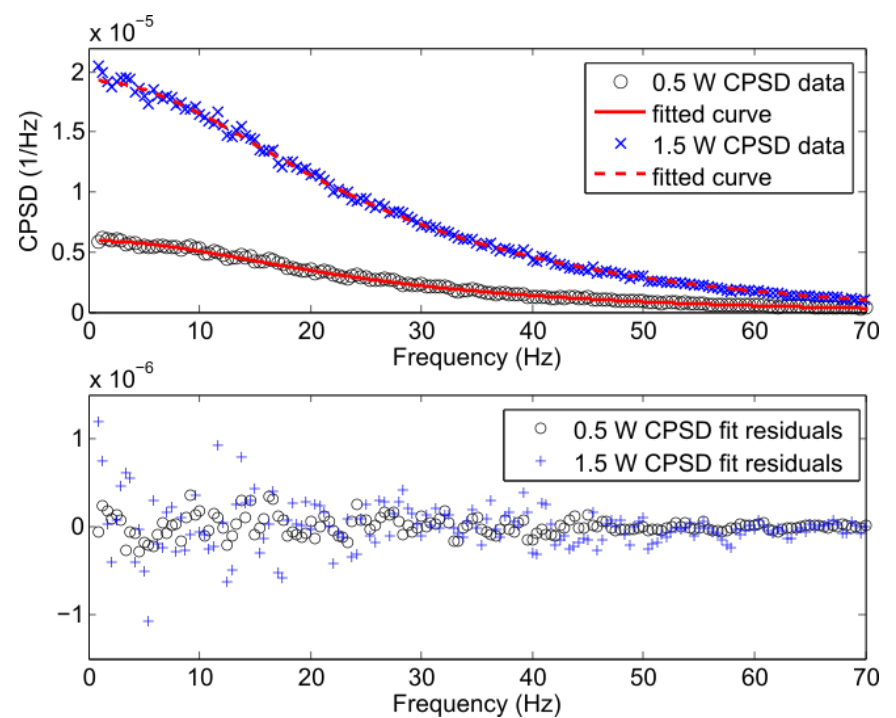

Fig. 4. Cross power spectral densities of the two compensated ionization chambers at critical states with powers of $0.5 \mathrm{~W}$ and $1.5 \mathrm{~W}$ with respective curve fits (top) and residuals (bottom).

\section{NOVEL CURRENT AMPLIFIER FOR FLEXIBLE DETECTOR USE}

\section{A. Design of current amplifier}

The CICs offer only a limited flexibility in terms of positioning as they are fixed to the grid and are relatively large. For a comparable or higher flexibility than with the $\mathrm{BF}_{3}$ detectors a current amplifier usable with, among others, Vniitfa ${ }^{3} \mathrm{He}$ ( 2.82 counts $\left./ \mathrm{n}_{\text {th }}\right)$ and Photonis CFUL01 ( 1 count $\left./ \mathrm{n}_{\text {th }}\right)$ detectors is being developed for this purpose. As the main purpose of the system is noise measurements, the set of specifications required can be summarized to be:

- Constant DC amplification in the range of $\mathrm{nA}$ to $\mathrm{mA}$ to allow for a simple acquisition system on FPGA basis.

- Constant transfer function in the region of interest for noise measurements i.e. $0 \mathrm{~Hz}$ to $\sim 200 \mathrm{~Hz}$.

The prototype board logic is depicted in Fig. 5. It consists of two main components:

i) A primary stage to pass through the high voltage to the detector's anode and to extract and amplify a current in the detector using a measurement resistance of $10 \mathrm{k} \Omega$ and an operational amplifier with a gain of 470 and a cut-off frequency of $2 \mathrm{kHz}$ at $-40 \mathrm{~dB} /$ decade. The current input range is hereby $2 \mathrm{pA}$ to $20 \mathrm{pA}$. Using a Photonis CFUL01 fission chamber, this results in an effective minimum measurable current in the chamber of $1 \mathrm{nA}$. In this configuration the measurement range in terms of reactor power is $0 \mathrm{~W}$ to $10 \mathrm{~W}$. An analog switch to augment the range in dependence of test results is foreseen for the final design.

ii) A secondary stage for active (doubled low-pass RC) filtering purposes with an effective gain of 0 and a cut-off frequency of $3.3 \mathrm{kHz}$ at $-25 \mathrm{~dB} /$ decade. Hereafter a BNC output connector gives the signal to acquisition hardware.

The transfer function of the prototype was measured and is depicted in Fig. 6. Indeed, in the range of interest for neutron noise measurements $(1-100 \mathrm{~Hz})$ the transfer function exhibits a plateau.

\section{B. Current mode neutron noise measurements in CROCUS using the new amplifier}

The new current amplifier was connected to a Photonis CFUL01 fission chamber as depicted in Fig. 5. set in the eastern reflector of the CROCUS core (see Fig 2.). The Plexiglas channel for the fission chamber is clamped to the grid and allows for flexible mounting in various locations in the reflector zone. We measured the output signal again using the Teledyne

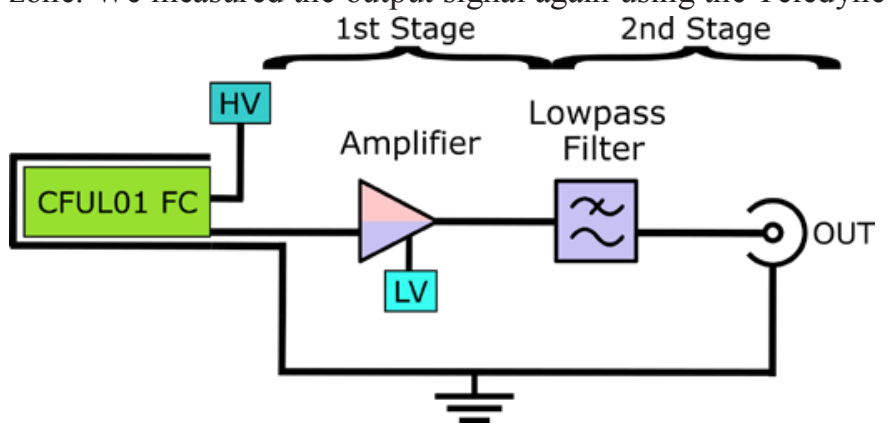

Fig. 5. Schematic of the board logic of the new current amplifier for noise measurements. The high voltage (HV) is passed through and the resulting measured detector current is amplified in the first stage. The second stage provides filtering and the output signal. Low voltage (LV) for the operational amplifiers is provided from an external source. 


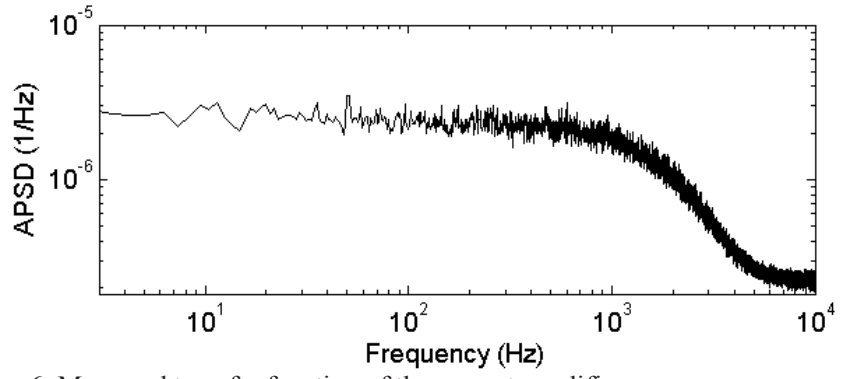

Fig. 6. Measured transfer function of the current amplifier.

Lecroy Wavesurfer 10 oscilloscope at a DC $10 \mathrm{M} \Omega$ input coupling. At this prototype stage, only one detector was used. A measurement time of $33 \mathrm{~min}$ at $100 \mathrm{~mW}$ was chosen as a first reference state. The signal was then treated via the MATLAB routine described in Section III B.

\section{Experimental results for new current station noise measurements}

The APSD was fitted following Equation 3 to determine the prompt decay constant (see Fig 7). The final value for the APSD estimate for $\alpha$ at $100 \mathrm{~mW}$ is $\mathbf{( 1 6 0 . 8} \pm \mathbf{6 . 3}) \mathbf{~ s}^{-1}$, and is in the same agreement range as the CIC measurement. The higher statistical uncertainty is attributed to the APSD method. CPSD analyses could allow for further precision once more experimental channels and electronics have been manufactured. This first measurement proves nonetheless the capability of the new system and allows us to further pursue its development.

\section{CONCLUSION AND OUTLOOK}

Future experimental activities in the CROCUS zero power facility call for improved and more flexible neutron noise measurement methods. A previously developed system using $\mathrm{BF}_{3}$ detectors and gamma spectroscopy electronics was limited in detection rate and sensitivity. For this purpose, the possibility of current mode acquisition was explored.

Two ${ }^{10} \mathrm{~B}$ compensated ionization chambers, used as operational monitors, already output an analog current signal, which proved usable for such measurements. Using an oscilloscope and postprocessing via the CPSD method the prompt neutron decay constant $\alpha$, was determined to be $(\mathbf{1 5 6 . 8} \pm \mathbf{3 . 2}) \mathbf{s}^{-1}$ at $0.5 \mathrm{~W}$ and $(\mathbf{1 6 0 . 2} \pm \mathbf{3 . 5}) \mathrm{s}^{-1}$ at $1.5 \mathrm{~W}$ using the CICs. Additionally, the development status of a novel current amplifier for other available detectors was presented. Characteristics and first current mode noise measurements using a CFUL01 fission chamber were shown. Preliminarily measurements using one detector with the new system yielded, using APSD analysis,

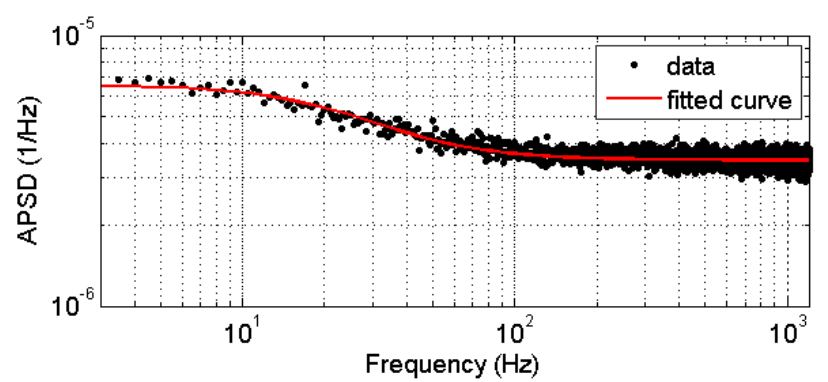

Fig. 7. Measured APSD using a Photonis CFUL01 fission chamber in the periphery of the CROCUS core connected to the new current amplifying system. $\mathbf{( 1 6 0 . 8} \pm \mathbf{6 . 3}) \mathbf{s}^{-1}$. All results are within $1 \sigma$ agreement with Monte Carlo predictions and previous measurements.

Neutron noise measurements in current mode allowed for shorter measurement durations as well as measurements at higher detection rates and thus meet the requirements for future experimental needs. Future improvements include a full characterization and validation of the new system using more detectors examining the full range of operation. This will allow for more cross correlation measurements and the calculation of $\beta_{\text {eff }}$ and $\Lambda$ by solving APSD/CPSD normalization.

The final design comprising versatile electronics as well as flexible detector positioning will then be used for experiments for spatial and spectral noise theory validation within CROCUS.

\section{ACKNOWLEDGMENT}

The authors thank D. Godat for his assistance in operating the reactor and with the mechanical aspects of detector positioning.

\section{REFERENCES}

[1] J.-M. Paratte, R. Früh, U. Kasemeyer, M.A. Kalugin, W. Timm, and R. Chawla. "A benchmark on the calculation of kinetic parameters based on reactivity effect experiments in the CROCUS reactor" Annals of Nuclear Energy, 33(8):739-748, May 2006.

[2] V. Roland, G. Perret, G. Girardin, P. Frajtag, and A. Pautz, "Neutron noise measurement in the CROCUS reactor," in Proc. IGORR, 2013.

[3] G. Perret, G. Girardin, P. Frajtag, M. Hursin, 2014. Decay constant and delayed neutron fraction measurements in CROCUS - TM-41-14-02 Rev. 1. Tech. rep., Paul Scherrer Institut, Villigen, 5232, Switzerland.

[4] G. Perret, B. Geslot, A. Gruel, P. Blaise, J. Di-Salvo, G. De Izarra, \& A. Pautz. (2017). Kinetic parameter measurements in the MINERVE reactor. IEEE Transactions on Nuclear Science, 64(1), 724-734.

[5] V. Lamirand, M. Hursin, P. Frajtag, A. Pautz, G. Perret \& O. Pakari (2016). Future experimental programmes in the CROCUS reactor. In Conference proceedings of RRFM/IGORR 2016 (No. EPFL-CONF218310, pp. 284-292).

[6] J. R. Sheff, \& R. W. Albrecht, (1966). The Space Dependence of Reactor Noise I-Theory. Nuclear Science and Engineering, 24(3), 246-259.

[7] G. F. Knoll. Radiation detection and measurement. Wiley \& Sons, 2000.

[8] C. E. Cohn, "A simplified theory of pile noise," Nucl. Sci. Eng., vol. 7, no. 5 , pp. $472-475,1960$.

[9] R. E. Uhrig. Random Noise Techniques in Nuclear Reactor Systems. Ronald Press, 1970.

[10] M. M. R. Williams. Random Processes in Nuclear Reactors. Pergamon Press, Oxford, 1974.

[11] G. de Izarra, C. Jammes, B. Geslot, J. Di Salvo, C. Destouches SPECTRON, a neutron noise measurement system in frequency domain Rev. Sci. Instrum., $86 \quad$ (11) (2015), p. 115111 http://scitation.aip.org/content/aip/journal/rsi/86/11/10.1063/1.4935250

[12] Teledyne Lecroy Wavesurfer 10 Datasheet, Accessed 9. May 2017: http://cdn.teledynelecroy.com/files/pdf/wavesurfer10-datasheet.pdf

[13] B. Diven, H. Martin, R. Taschek, and J. Terrell, "Multiplicities of Fission Neutrons," Phys. Rev., vol. 101, no. 3, pp. 1012-1015, Feb. 1956

[14] Harris, Fredric J. "On the use of windows for harmonic analysis with the discrete Fourier transform." Proceedings of the IEEE 66.1 (1978): 51-83. 